\title{
Optimization of an organic memristor as an adaptive memory element
}

\author{
Tatiana Berzina, ${ }^{1,2}$ Anteo Smerieri, ${ }^{1}$ Marco Bernabò, ${ }^{3}$ Andrea Pucci, ${ }^{3}$ Giacomo Ruggeri, ${ }^{3}$ \\ Victor Erokhin, ${ }^{1,2, a)}$ and M. P. Fontana ${ }^{1,2}$ \\ ${ }^{1}$ Department of Physics, University of Parma, Viale Usberti 7A, Parma 43100, Italy \\ ${ }^{2}$ CRS SOFT CNR-INFM, Rome 00185, Italy \\ ${ }^{3}$ Department of Chemistry and Industrial Chemistry, University of Pisa, Via Risorgimento 35, \\ Pisa 56126, Italy
}

(Received 9 April 2009; accepted 14 May 2009; published online 24 June 2009)

\begin{abstract}
The combination of memory and signal handling characteristics of a memristor makes it a promising candidate for adaptive bioinspired information processing systems. This poses stringent requirements on the basic device, such as stability and reproducibility over a large number of training/learning cycles, and a large anisotropy in the fundamental control material parameter, in our case the electrical conductivity. In this work we report results on the improved performance of electrochemically controlled polymeric memristors, where optimization of a conducting polymer (polyaniline) in the active channel and better environmental control of fabrication methods led to a large increase both in the absolute values of the conductivity in the partially oxydized state of polyaniline and of the on-off conductivity ratio. These improvements are crucial for the application of the organic memristor to adaptive complex signal handling networks. (c) 2009 American Institute of Physics. [DOI: 10.1063/1.3153944]
\end{abstract}

\section{INTRODUCTION}

The memristor, a device which may be considered as a resistor with memory (i.e., the resistance value depends on its previous history), was postulated many years ago, ${ }^{1}$ but only recently practical realizations have been reported. In particular, a recent work describes a completely inorganic nanoscale device in which the memory effect was explained by the dopant redistribution after the voltage application. ${ }^{2}$ However, a more reliable explanation of the memory effect may involve redox reactions in the active conduction medium. ${ }^{3}$

Redox reactions have been used as a working principle for several organic electronic devices. In particular, electrochemical field-effect transistors have been extensively studied for more than 20 years. ${ }^{4}$ The rectifying behavior observed for an electrochemical junction realized with a solid electrolyte ${ }^{5}$ can be considered as a further step toward the element with memory.

Some years ago we have reported a molecular electronic device, based on the electrochemical control of resistivity of a conducting polymer, which exhibited interesting electrical characteristics. ${ }^{6}$ Even if its construction seems to be rather similar to traditional electrochemical field-effect transistors, its working principle is actually closer to that of the electrochemical rectifier. ${ }^{5}$ In particular, all conductivity variations occur in the restricted active area of a conducting polymer film in contact with a solid electrolyte. For this heterojunction we have demonstrated several unusual electrical properties, such as a memory effect of the device resistance, ${ }^{6}$ which implies that it can be considered as a first example of a totally organic memristor, and current auto-oscillation

\footnotetext{
${ }^{\text {a) }}$ Author to whom correspondence should be addressed. Tel.: +390521 905235. FAX: +39 0521 905223. Electronic mail: victor.erokhin@fis.unipr.it.
}

generation. ${ }^{7}$ Successive studies have indicated that the device could be used as a main node in adaptive signal handling networks, ${ }^{8}$ mimicking to some extent the learning behavior of biological systems. ${ }^{9}$ Further development of this work for the realization of really complex networks capable of information processing and adaptive learning could open quite interesting perspectives. In particular, a critical feature of such systems would be the fact that the memory and information processing functions would be performed by the same elements. Previous experience of the system will modify the properties of the constituting elements making it more adequate for the solving of new similar problems. Such behavior is the basis of synaptic learning, i.e., learning governed by Hebbian rule. ${ }^{10}$ Similar processes are deemed to occur in biological organisms, where in many cases learning can be considered as the variation of the synaptic weight functions, which can vary the signal propagation pathways in the nervous system. ${ }^{11}$

The working principle of our device is based on the difference in the conductivity of a conducting polymer, namely, polyaniline (PANI) in the oxidized and reduced states. ${ }^{12} \mathrm{~A}$ detailed description of the device can be found in Ref. 6. The device is schematically shown in Fig. 1.

A thin PANI layer, used as a conducting channel, is de-

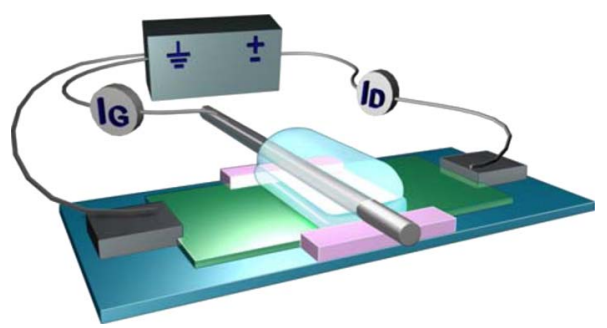

FIG. 1. (Color online) Schematic representation of the organic memristor with external power supply and measuring devices. 
posited onto an insulating solid support with two metal electrodes. A stripe of solid electrolyte is deposited in the central part of the channel. The PANI area under the electrolyte is the active zone, as the oxidation and reduction in PANI take place in this area. Of course, some redox reactions can occur also outside this active zone due to possible diffusion. However, the length of diffusion must be negligible with respect to the active zone width. In fact, micro-Raman investigation of the PANI state map as the device was cycled has demonstrated with micron resolution that all redox transformations take place within the active zone. ${ }^{13}$ We expect, however, that the diffusion length is even significantly less than the resolution. The analysis of the kinetics of the conductivity transformations has shown that it requires about $20 \mathrm{~min}$ for arriving to the saturation [diffusion to the whole PANI depth that is about $50 \mathrm{~nm}$ (Ref. 6)]. Considering the working time values in the present work, we can estimate the length as several nanometers that is really negligible with respect to the active zone width (about $1 \mathrm{~mm}$ ). A silver wire is attached to the electrolyte layer to be used as a reference point. In the normal configuration the reference (gate) electrode is connected to the source and both of them are connected to the ground potential level. Thus, the reference potential is zero and reduction or oxidation takes place according to the actual potential of the active zone. Even if the device is connected to the circuit by two points only, two different currents, namely, the ionic one in the electrolyte and the total current passing through the device, were measured and considered for better understanding of the device functioning. This by the way makes our device closer to the originally proposed memristor. ${ }^{1}$

The possibility of using such devices in adaptive networks is determined by their property to increase their conductivity after application of a positive voltage higher than a certain value (usually +0.6 to $+1.0 \mathrm{~V}$ depending on the particular construction of the device) and its decrease after application of any negative voltage. The time constants of these variations are different: about one order of magnitude difference in time constants necessary for saturating processes. Thus, unsupervised learning in the network will take place due to the increase in the conductivity of the elements located along pathways more frequently used for the signal transmission. Supervised learning, instead, will be performed by negative voltage application between input-output pairs connected by preferential pathways, formed during unsupervised learning of the system, but that are considered as $a$ priori wrong. Such negative voltage application will inhibit the conductivity of elements along this pathway.

Simple circuits demonstrating the possibility of both supervised $^{8}$ and unsupervised ${ }^{9}$ learning of networks based on these organic memristors have been already realized. However, real complex (up to decision making) signal processing demands the formation of networks including a significantly larger number of such elements working for a rather long time (i.e., over thousands of learning cycles), necessary for effective learning. Thus, there are several requirements to memristor properties that must be satisfied before such networks could be realized. First of all, the conductivity ratio in the oxidized conducting and reduced insulating states must be as high as possible. In the previously realized elements there the ratio was about 100 . Second, the absolute value of the conductivity of the memristor in the oxidized state must be rather high. In fact, if the signal pathways in the network contain thousands of such elements, high conductivity will guarantee a high signal-to-noise ratio level. Finally, it is very important to have high stability of the memristor properties. In the framework of the adaptive networks, the term "stability" has not the same meaning as for materials in traditional electronics, where it refers to the constant behavior of the functioning element in time. In our case instead the element must vary its electrical properties according to its previous history or due to the external training. Thus, we must consider some other parameter as an indication of the element stability. For this we consider the ability of the element to reproduce electrical properties' variation during multiple cyclic applications of variable potentials.

From this point of view we have already shown that the properties of the element realized on the basis of commercially available materials are rather stable for more than 4000 min and can allow at least 50 adaptations. ${ }^{14}$ Such stability permits the construction of networks capable to adaptations 8 and mimicking learning of simple biological benchmarks; ${ }^{9}$ however, for complex signal processing, the stability must be increased.

The aim of this work is to investigate the influence of the channel composition and doping on the properties of the memristor with special emphasis on the three items described above, namely, conductivity ratio in reduced and oxidized states, absolute value of the conductivity in the oxidized state, and the stability of the device properties. For this last item, in particular, a new device configuration was developed.

\section{MATERIALS AND METHODS}

Aniline (Aldrich, 99.5+\% ACS (American Chemical Society specification of purity grade)) was distilled under $\mathrm{CaH}_{2}$ at reduced pressure and stored in argon atmosphere. Dodecyl benzene sulfonic acid (DBSA) (used for doping) (Fluka, 90\% tech. isomers' mixture), ammonium persulfate (APS) (Fluka, 98\% ACS (American Chemical Society specification of purity grade), and $N$-methyl pyrrolidone (1-methyl-2pyrrolidinone, Aldrich, spectrophotometric grade, $\geq 99 \%$ ) were used as received. Water HPLC (purity grade for High Performance Liquid Chromatography) grade was used.

PANI was prepared according to literature. ${ }^{15,16}$ Briefly, in a two-necked flask equipped with a mechanical stirrer and a dropping funnel, $14.40 \mathrm{~g}$ of DBSA dissolved in $800 \mathrm{ml}$ of water and $4 \mathrm{~g}$ of aniline were gently added. The mixture was stirred for $3 \mathrm{~h}$ at room temperature until formation of the typical milklike solution of the anilinium salt and then the solution was cooled to $0{ }^{\circ} \mathrm{C}$ by dipping the flask in a waterice bath. Successively, five drops of a saturated solution of cobalt sulfate were added to the anilinium salt solution and then $10 \mathrm{~g}$ of APS dissolved in $35 \mathrm{ml}$ of water were slowly dropped to the solution. After $5 \mathrm{~h}$ the stirring was stopped and the solution containing a blue-dark precipitate was stored for a night at room temperature. The precipitate was 


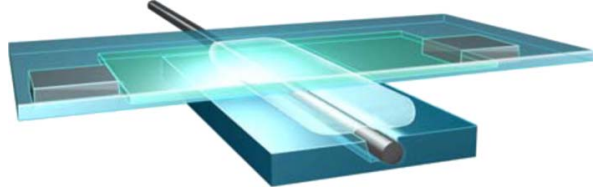

FIG. 2. (Color online) Scheme of the element with sandwich structure.

collected by adding 11 of methanol to the suspension and by filtering it with a Buchner filter. Finally the solid powder was repeatedly washed with methanol and water until neutrality. The PANI was then dried several days under vacuum pump.

Infrared spectra [Fourier transform infrared (FTIR)] were performed with a Fourier transform spectrometer "PerkinElmer" Spectrum One" on films cast from $N$-methyl pyrrolidone solution on a $\mathrm{KBr}$ window. A list of the characteristic absorption bands studied is given as follows: FTIR $\left(\mathrm{cm}^{-1}\right)$ : $3450\left(\nu \mathrm{N}-\mathrm{H}, \mathrm{NH}_{2-}\right) ; 3236(\nu \mathrm{N}-\mathrm{H}) ; 2956\left(\nu_{\text {as }} \mathrm{C}-\mathrm{H}\right.$, $\left.\mathrm{CH}_{3^{-}}\right) ; 2924\left(\nu_{\mathrm{as}} \mathrm{C}-\mathrm{H}, \mathrm{CH}_{2^{-}}\right) ; 2851\left(\nu_{\mathrm{S}} \mathrm{C}-\mathrm{H}, \mathrm{CH}_{2^{-}}\right) ; 1557(\nu$ $\mathrm{N}=\mathrm{Q}=\mathrm{N}) ; 1469(\nu \mathrm{N}-\mathrm{B}-\mathrm{N}) ; 1384\left(\nu \mathrm{C}-\mathrm{N}, \mathrm{QB}_{t} \mathrm{Q}\right) ; 1296(\nu$ $\left.\mathrm{C}-\mathrm{N}, \mathrm{QB}_{c} \mathrm{Q}, \mathrm{QBB}, \mathrm{BBQ}\right) ; 1233,1256(\nu \mathrm{C}-\mathrm{N}, \mathrm{BBB}) ; 1109$ $\left(\delta \mathrm{N}=\mathrm{Q}=\mathrm{N}\right.$ and $\left.\mathrm{Q}=\mathrm{NH}-\mathrm{Bo} \mathrm{B}-\mathrm{NH}^{+}-\mathrm{B}\right)$; and 1032 .

Elemental analyses (EAs) were accomplished by the microanalysis laboratory at the Faculty of Pharmacy, University of Pisa.

EA (\%, found): C 69.1; H 7.6; N 5.8; S 7.7.

Thermogravimetric scans were obtained with a Mettler Toledo Starc System moduloTGA/SDTA851 ${ }^{\mathrm{e}}$ under nitrogen flux, at a scan rate of $20{ }^{\circ} \mathrm{C} / \mathrm{min}$.

TGA $\left({ }^{\circ} \mathrm{C}\right.$, onset): 335 .

UV-vis absorption spectra of polymer films were recorded under isotropic conditions with a Perkin-Elmer Lambda 650 on films cast from $N$-methyl pyrrolidone solution on quarts slide.

UV-vis (nm): 450, 800.

A special feature of such preparation is that the material is already partially doped before the beginning of the device construction.

Polyethylene oxide (8.000.000 Da) from Sigma with the concentration of $20 \mathrm{mg} / \mathrm{ml}$ doped with $0.1 M \mathrm{LiClO}_{4}$ was used as a solid electrolyte.

The active channel of the device was prepared by the deposition of 48 layers of PANI onto a glass substrate with two evaporated $\mathrm{Cr}$ electrodes, using the Langmuir-Schaefer (horizontal dipping) technique. The multilayer was additionally doped by $\mathrm{HCl}$ treatment. Deposition and doping procedures were similar to those described in Ref. 17.

About $1 \mathrm{~mm}$ in width stripe of Polyethylene oxide (PEO) ( 8.000.00 Da) was deposited in the central zone of the channel by solution casting. A $0.05 \mathrm{~mm} \mathrm{Ag}$ wire was attached to the PEO stripe. The completed structure was additionally doped in $\mathrm{HCl}$ vapor.

An alternative "sandwich" structure for the device was tried. In this case the assembling procedure illustrated in Fig. 2 was as follows.

A PANI channel was formed on a glass support with two electrodes (upper plate in Fig. 2) similarly to the previous case. A groove with a depth corresponding to the silver wire diameter was formed in the other solid support (glass). The

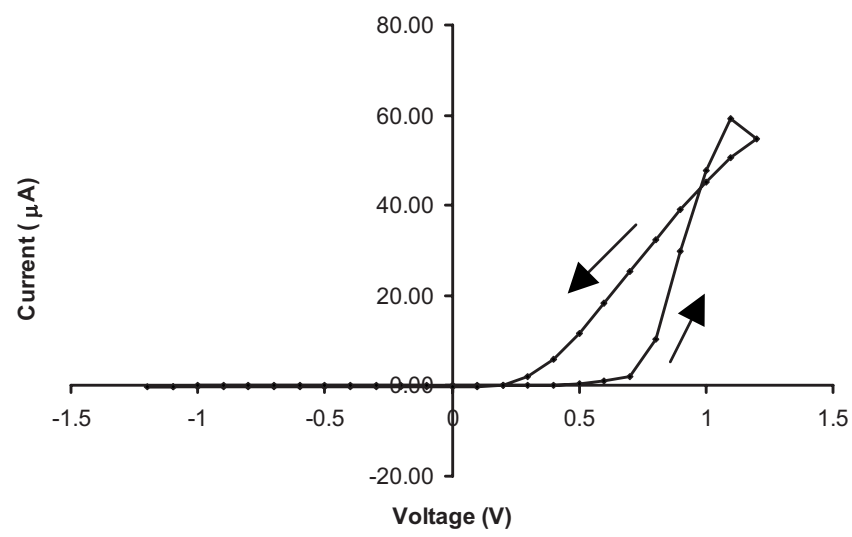

FIG. 3. Cyclic voltage-current characteristics of the organic memristor. The arrows indicate the direction (increase or decrease) of the voltage scan.

reference wire was placed into this groove and covered by a PEO solid electrolyte (bottom plate in Fig. 2). After drying for $30 \mathrm{~min}$ these two supports with formed structures were put in contact mechanically to form the device shown in Fig. 2.

Electrical measurements were performed as follows: The source and gate electrodes were always at ground potential; the drain potential was swept between -1.2 and $+1.2 \mathrm{~V}$, with steps of $0.1 \mathrm{~V}$. Each sweep was started with a drain potential of $0 \mathrm{~V}$, increasing it up to $+1.2 \mathrm{~V}$, then decreasing it until $-1.2 \mathrm{~V}$ and finally increasing it back to $0 \mathrm{~V}$. A $1 \mathrm{~min}$ delay was used after application of each voltage value and before the current reading, to allow the device to stabilize itself to the new conditions. Voltage sweep and drain current measurements were performed with a 236 source measure unit (Keithley), while gate current measurements were performed with a 6514 system electrometer (Keithley). Both Keithley units were linked to a PC and operated via MATLAB scripts, which allowed full automation of the measuring procedure.

Initially, measurements were performed at ambient conditions. Measurements in a controlled environment were performed in a closed plastic chamber (about 1.51 volume) with dried atmosphere and residual $\mathrm{HCl}$ vapor (about $10^{-4} \mathrm{M}$, corresponding to a partial pressure of about $200 \mathrm{~Pa}$ ).

\section{RESULTS AND DISCUSSION}

The cyclic voltage-current characteristics of the device is shown in Fig. 3.

The characteristics show a pronounced rectifying behavior with hysteresis. In the initial stage of the cycle (low positive potentials) the active channel is in the insulating form. The transition to the conductive state takes place at the potential of about $+0.6 \mathrm{~V}$ in good agreement with our previous measurements when PANI was doped only with $\mathrm{HCl}^{6}{ }^{6}$ In the back scan of the voltage the device has high conductivity with practically Ohmic behavior. Its transition to the insulating state takes place at about $0.1 \mathrm{~V}$ of the drain voltage.

From the behavior of the characteristics we can conclude that the two critical requirements for the memristor electrical properties, mentioned in the Introduction, are satisfied. In fact, the device has very high conductivity in the conductive 


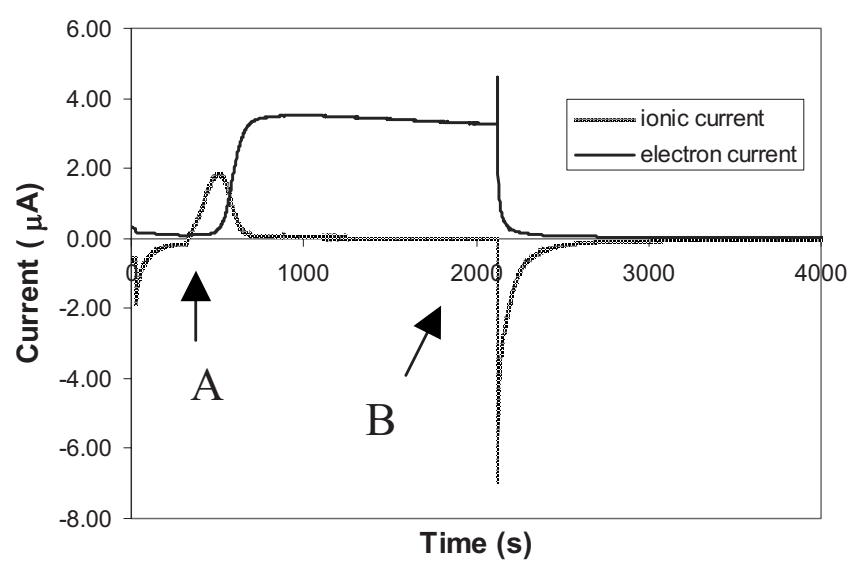

FIG. 4. Temporal behavior of the electron and ionic currents of the organic memristor for positive $(+0.6 \mathrm{~V}$; point $A)$ and negative $(-0.2 \mathrm{~V}$, point $B)$ drain voltages.

state. The reached current value is more than $60 \mu \mathrm{A}$ (at a voltage of $1.0 \mathrm{~V}$ ), which is much higher with respect to our previous results $(1.0-2.0 \mu \mathrm{A})$; furthermore, the conductivity ratio of the device in the conducting and insulating states, which is probably even more important for the realization of the adaptive networks, jumped from about 100 for the positive and negative branches of the cyclic voltage-current characteristics to about 2000 in the present device. This improvement of more than one order of magnitude is a very encouraging result. The kinetics of the conductivity variation is also an important characteristic of the device. The temporal behavior of the ionic (gate) and electron (drain minus gate) currents through the device for positive and negative potentials is shown in Fig. 4.

From a comparison with our previous results ${ }^{6}$ we see that the time constant of the conductivity variation in the case of the negative potential is similar, while for the positive voltage it becomes faster. This last finding can be due to the increased conductivity ratio for two different states of the PANI channel. In fact, the slower kinetics for the positive potential was explained by the gradual oxidation of the PANI in the active zone and continuous spatial redistribution of the potential along its length. If the conductivity anisotropy is increased, such redistribution should occur faster. In order to check this hypothesis we have applied the model ${ }^{18}$ that was successfully used to interpret not only differences in the conductivity variation kinetics for negative and positive polarities but also the appearance of auto-oscillations of the current for the device capable for the charge accumulation. ${ }^{7}$ Within the model, the active zone is divided in narrow stripes with its own timer. Conductivity variations take place within each stripe after passing reduction or oxidation potential with respect to the reference electrode according to the kinetics, experimentally found for the negative bias. The program recalculates electronic and ionic currents, and voltage distribution profile along the PANI channel after each time intervals. The results of the simulations are shown in Fig. 5 for the device with standard properties (b) and with those observed in the present case (a), i.e., increased difference in conductivities for oxidized and reduced states and with increased ionic conductivity.

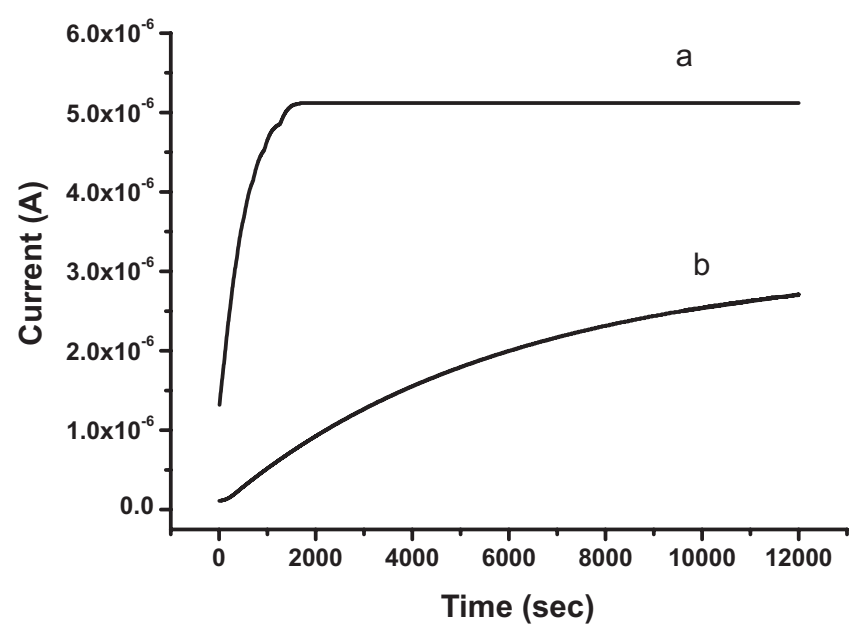

FIG. 5. Simulations of the conductivity variation for the device biased with $+0.6 \mathrm{~V}$ according to the model described in Ref. 10. (a) Device with increased difference of the conductivity in oxidized and reduced states (two orders of magnitude higher than in previous case) and with increased ionic conductivity (one order of magnitude higher than in previous case). (b) Device with previously (Ref. 2) described properties.

However, multicycle investigation of the device properties has revealed that the stability of the electrical properties was similar to those for the element with a standard channel. ${ }^{14}$ The degradation of the properties was attributed previously to the partial dedoping of the PANI layer. Therefore, further measurements were performed in the chamber with controlled $\mathrm{HCl}$ partial pressure. The temporal behavior of the drain current during the application of cyclic scans of the drain voltage is shown in Fig. 6.

Logarithmic dependence of the peak current value on the number of cycle is shown in Fig. 7. The absolute value of the device conductivity decreases exponentially for the first 5 cycles and then becomes practically constant after the tenth cycle, remaining still very high with respect to even freshly prepared samples measured in the ambient conditions. The initial decrease in the conductivity is a normal phenomenon for the conducting polymers, which can be due to several factors, such as some contact processes on the boundary with metal electrodes or redistribution of the dopant upon reaching equilibrium conditions. Thus, the conditions with residual acid vapor can improve the performance of the device.

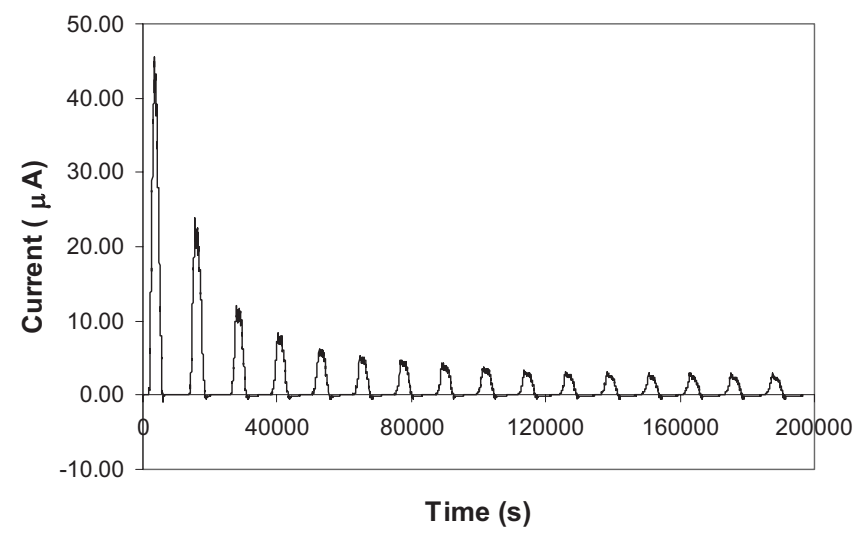

FIG. 6. Temporal behavior of the drain current during multicycle application of the drain voltage. 


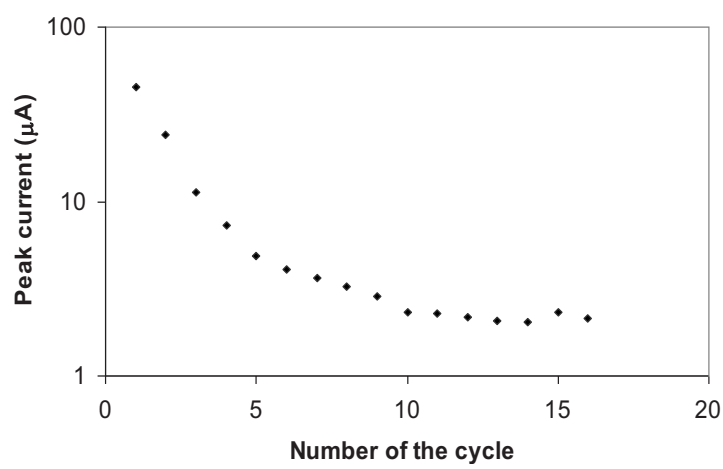

FIG. 7. Logarithmic dependence of the peak current on the number of the cycle.

However, an element that does not demand the use of special measuring chambers would be more practical. A solution could be to prevent dedoping by appropriate sealing. The first step in this direction was performed by assembling the device in a sandwich configuration (Fig. 2). Confinement of the active zone between two solid plates was expected to make more difficult the dedoping of PANI. The results of the drain current temporal behavior during multicyclic application of the drain voltage were found to be similar to those obtained in controlled conditions (Fig. 6). We have observed the rather significant decrease in the maximum drain current for first cycles. However, after 5 cycles, the characteristics were well stabilized. Cyclic voltage-current characteristics for cycles 6-8 are shown in Fig. 8 .

As it is clear from Fig. 8, such sealing has resulted in the significant improvement of the device properties' stability, which persisted for many successive cycles.

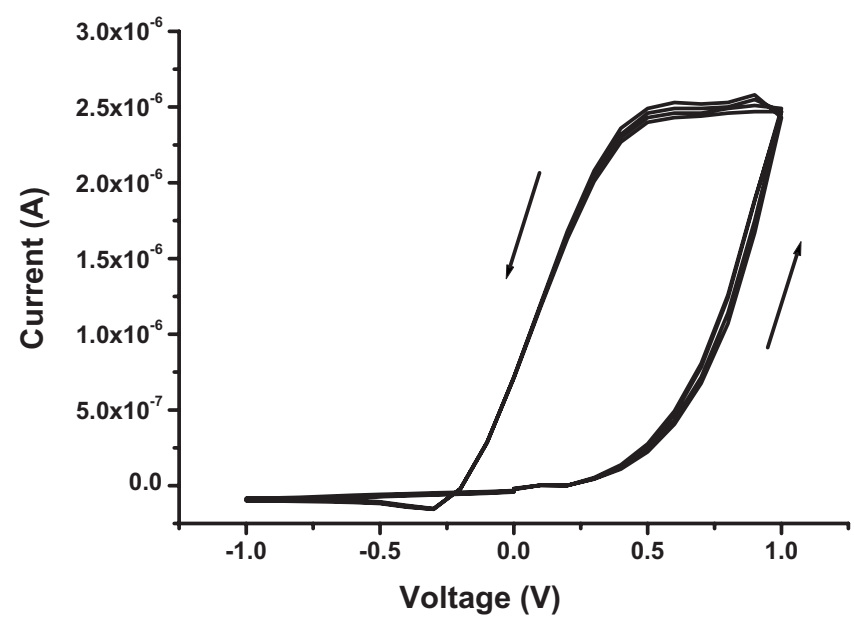

FIG. 8. Cyclic voltage-current characteristics for a sandwich structure for the fifth, sixth, and seventh cycles. The arrows indicate the direction (increase or decrease) of the voltage scan.

\section{CONCLUSIONS}

Utilization of PANI, predoped with a rather strong acid (DBSA), resulted in the improvement of two important parameters of the polymeric electrochemical device (organic memristor), namely, the conductivity ratio in conducting and insulating states and in the absolute value of the conductivity for the positive polarity of the applied voltage. Due to the presence of the high molecular weight anion, the use of DBSA during the PANI synthesis guarantees the significant decrease in the dedoping rate, which is rather high in the case of $\mathrm{HCl}$ doping, resulting in the improvement of the device characteristics and its working time. This result is very important for the successful realization of the adaptive networks based on a large number of such elements. We can expect, in fact, a high signal-to-noise ratio and possibility of effective supervised and unsupervised learning of the system. The increase in the stability of electrical properties' variation can be obtained by measurements in controlled conditions or by effective sealing of the fabricated structures.

\section{ACKNOWLEDGMENTS}

We acknowledge the financial support of the Future and Emerging Technologies (FET) programme within the Seventh Framework Programme for Research of the European Commission, under the FET-OPEN grant agreement BION, Grant No. 213219.

${ }^{1}$ L. O. Chua, IEEE Trans. Circuit Theory 18, 507 (1971).

${ }^{2}$ D. B. Strukov, G. S. Snider, D. R. Stewart, and R. S. Williams, Nature (London) 453, 80 (2008).

${ }^{3}$ J. Wu and R. L. McCreery, J. Electrochem. Soc. 156(1), P29 (2009).

${ }^{4}$ C. F. Shu and M. S. Wrighton, J. Phys. Chem. 92, 5221 (1988).

${ }^{5}$ M. Chen, D. Nilsson, T. Kugler, and M. Berggren, Appl. Phys. Lett. 81, 2011 (2002).

${ }^{6}$ V. Erokhin, T. Berzina, and M. P. Fontana, J. Appl. Phys. 97, 064501 (2005).

${ }^{7}$ V. Erokhin, T. Berzina, P. Camorani, and M. P. Fontana, J. Phys.: Condens. Matter 19, 205111 (2007).

${ }^{8}$ V. Erokhin, T. Berzina, and M. P. Fontana, Crystallogr. Rep. 52, 159 (2007).

${ }^{9}$ A. Smerieri, T. Berzina, V. Erokhin, and M. P. Fontana, Mater. Sci. Eng., C 28, 18 (2008).

${ }^{10}$ D. O. Hebb, The Organization of Behavior: A Neuropsychological Theory, 2nd ed. (Wiley, New York, 1961).

${ }^{11}$ P. R. Benjamin, K. Staras, and G. Kemenes, Learn. Memory 7, 124 (2000).

${ }^{12}$ E. T. Kang, K. G. Neoh, and K. L. Tan, Prog. Polym. Sci. 23, 277 (1998).

${ }^{13}$ T. Berzina, V. Erokhin, and M. P. Fontana, J. Appl. Phys. 101, 024501 (2007).

${ }^{14}$ V. Erokhin, T. Berzina, P. Camorani, and M. P. Fontana, Colloids Surf., A 321, 218 (2008).

${ }^{15}$ Y. Haba, E. Segal, M. Narkis, G. I. Titelman, and A. Siegmann, Synth. Met. 106, 59 (1999).

${ }^{16}$ W. A. Gazotti and M.-A. De Paoli, Synth. Met. 80, 263 (1996).

${ }^{17}$ V. I. Troitsky, T. S. Berzina, and M. P. Fontana, Synth. Met. 129, 39 (2002).

${ }^{18}$ A. Smerieri, V. Erokhin, and M. P. Fontana, J. Appl. Phys. 103, 094517 (2008). 